\title{
Global Dynamics of an SEIR Model with Infectious Force in Latent and Recovered Period and Standard Incidence Rate
}

\author{
Yanli $\mathrm{Ma}^{*}$ \\ Department of Common Course, Anhui Xinhua University, Hefei Anhui 230088, China. \\ * Corresponding author. Tel.: +86 5516519 3105; email: Linda-mayanli@163.com \\ Manuscript submitted March 30, 2016; accepted September 23, 2016. \\ doi: 10.17706/ijapm.2017.7.1.1-11
}

\begin{abstract}
In this paper, we consider an epidemic model with the infectious force in the latent and recovered period and establish the SEIR epidemic model with standard incidence rate. Then, we find the basic reproduction number $R_{0}$ which determines whether the disease exists. By using Liapunov function method, we prove that the disease-free equilibrium $E_{0}$ is globally asymptotically stable and the disease goes away when $R_{0}<1$. By Hurwitz criterion, we also prove that $E_{0}$ is unstable and the unique endemic equilibrium $E^{*}$ is locally asymptotically stable when $R_{0}>1$. It is shown that when disease-induced death rate and elimination rate are zero, $E^{*}$ is globally asymptotically stable and the disease persists. Finally, we give numerical simulation to illustrate the theoretical analysis.
\end{abstract}

Key words: Basic reproductive number, equilibrium, stability, SEIR epidemic model, numerical simulation.

\section{Introduction}

In our life, there are a variety of infectious diseases. For a long time mathematical models which describe the population dynamics of infectious diseases have been playing an important role in a better understanding of disease control and epidemic pattern. In order to predict the spread of infectious disease among the areas, the transmission dynamics of infectious disease is studied by many epidemic models in host populations. The dynamics of the classic SIR or SIRS epidemic models have been widely studied [1]-[10]. However, many infectious diseases such as pertussis, SARS and so on, incubate inside the population for a period of time before they become infectious. So the systems that are more general than SIR or SIRS types need to study the role of incubation in the spread of infectious disease. The present model is of SEIR or SEIRS class, depending on whether the adaptive immunity is permanent or otherwise.

Li [11] analyzed the global dynamics of a SEIR model with varying total population size. Fan [12] discussed the global stability of an SEIS epidemic model with recruitment and a varying total population size. Sun [13] studied the global analysis of an SEIR model with varying population size and vaccination. Yi [14] considered an SEIR epidemic system with nonlinear transmission rate. Zhang [15] studied global asymptotic stability of a delayed SEIRS epidemic model with saturation incidence. Yan and Zhang [16] considered an SEIR epidemic system with nonlinear transmission rate. Liu [17] analyzed the global stability of an SEIR epidemic model with age-dependent latency and relapse.

However, for malaria and some other infectious diseases, the latent and immune period may be infectious. The epidemic model of SEIR with infectious force in both infected and recovered period is rarely studied in the paper. Motivated by literature [11]-[17], we consider an epidemic model with the infectious force in the 
latent and recovered period and establish the SEIR epidemic model with standard incidence rate.

\section{Model Formulation}

The host population is divided into four classes, the susceptible, latent, infectious, and recovered, with sizes denoted by $S(t), E(t), I(t)$ and $R(t)$, respectively. The SEIR model having infectious force in the latent and recovered period is depicted in the following system of differential equations

$$
\left\{\begin{array}{l}
S^{\prime}=A-\lambda_{1} \frac{S E}{N}-\lambda_{2} \frac{S I}{N}-\lambda_{3} \frac{S R}{N}-d S, \\
E^{\prime}=\lambda_{1} \frac{S E}{N}+\lambda_{2} \frac{S I}{N}+\lambda_{3} \frac{S R}{N}-\left(\gamma+d+\alpha_{1}+k_{1}\right) E, \\
I^{\prime}=\gamma E-\left(\varepsilon+d+\alpha_{2}+k_{2}\right) I, \\
R^{\prime}=\varepsilon I-d R .
\end{array}\right.
$$

where $A$ is constant recruitment rate of the population;, $\lambda_{2}, \lambda_{3}$ are the rate of the efficient contact in the latent, infected and recovered period respectively; $\alpha_{1}, \alpha_{2}$ are denote the rate of disease-caused death of the exposed and the infectious respectively; $d$ is the natural death rate of the population; $k_{1}, k_{2}$ are the elimination rate of the exposed and the infectious respectively; $\gamma$ is the transfer rates between the exposed and the infectious; $\varepsilon$ is the removed rate from the infective class to the recovered class.

The total population size $N(t)$ which can be determined by $N(t)=S(t)+E(t)+I(t)+R(t)$ implies $N^{\prime} \leq A-d N$. From biological considerations, we study (1) in the feasible region $\Gamma=\left\{(S, E, I, R) \in R_{+}^{4} \mid 0 \leq S+E+I+R=N \leq A / d\right\}$, where $R_{+}^{4}$ denotes the non-negative cone and its lower dimensional faces. $\Gamma$ can be shown to be positively invariant with respect to (1).

Set each of the differential equations on the right side equal to zero in (1), we have the following system

$$
\left\{\begin{array}{l}
S=\frac{N A((\delta-d) \omega-\gamma(d+\varepsilon))}{\left(\lambda_{1} \omega+\lambda_{2} \gamma+\lambda_{3} \frac{\varepsilon \gamma}{d}\right)(A-d N)+d N((\delta-d) \omega-\gamma(d+\varepsilon))} \\
E=\frac{\omega(A-d N)}{(\delta-d) \omega-\gamma(d+\varepsilon)} \\
I=\frac{\gamma(A-d N)}{(\delta-d) \omega-\gamma(d+\varepsilon)} \\
R=\frac{\frac{\varepsilon \gamma}{d}(A-d N)}{(\delta-d) \omega-\gamma(d+\varepsilon)}
\end{array}\right.
$$

And we have the following equation about $N$

$$
F(N)(A-d N)=0
$$


where

$$
F(N)=-A d\left(\lambda_{1} \omega+\lambda_{2} \gamma+\lambda_{3} \frac{\varepsilon \gamma}{d}\right)\left(\omega+\gamma+\frac{\varepsilon \gamma}{d}\right)+\delta \omega d N\left(\lambda_{1} \omega+\lambda_{2} \gamma+\lambda_{3} \frac{\varepsilon \gamma}{d}-((\delta-d) \omega-\gamma(d+\varepsilon))\right)
$$

so the system (1) always has the disease-free equilibrium $E_{0}(A / d, 0,0,0)$ in the interval $(0, A / d)$.

Define the basic reproduction number of (1) as $R_{0}=\frac{\lambda_{1} \omega+\lambda_{2} \gamma+\lambda_{3} \frac{\varepsilon \gamma}{d}}{\delta \omega}$. Since

$$
F(0)=-A d\left(\lambda_{1} \omega+\lambda_{2} \gamma+\lambda_{3} \frac{\varepsilon \gamma}{d}\right)\left(\omega+\gamma+\frac{\varepsilon \gamma}{d}\right)<0, F(A / d)=\delta \omega A(\delta \omega-d \omega-d \gamma)\left(R_{0}-1\right)
$$

$F(N)$ is monotone increasing and $F(A / d)>0$, so that $F(N)=0$ has only a positive root in $(0, A / d)$, (1) has an unique endemic equilibrium $E^{*}\left(S^{*}, E^{*}, I^{*}, R^{*}\right)$, where $S^{*}, E^{*}, I^{*}, R^{*}$ are determined by (2).

\section{Global Stability of the Disease-free Equilibrium}

Theorem 1. The disease-free equilibrium $E_{0}$ of (1) is globally asymptotically stable in $\Gamma$ if $R_{0} \leq 1$ and $E_{0}$ is unstable if $R_{0}>1$. The solutions to (1) starting sufficiently close to $E_{0}$ in $\Gamma$ move away from $E_{0}$ except that those starting on the invariant $\mathrm{S}$ axis approach $E_{0}$ along this axis.

Proof. Constructing a Liapunov function $V=\frac{\lambda_{1} \omega+\lambda_{2} \gamma+\lambda_{3} \frac{\varepsilon \gamma}{d}}{\delta \omega} E+\frac{\lambda_{2}+\frac{\varepsilon}{d} \lambda_{3}}{\omega} I+\frac{\lambda_{3}}{d} R$,

Its derivative along the solutions of (1) with respect to $t$ gives

$$
\begin{gathered}
\left.\frac{d V}{d t}\right|_{(1)}=\frac{\lambda_{1} \omega+\lambda_{2} \gamma+\lambda_{3} \frac{\varepsilon \gamma}{d}}{\delta \omega}\left(\lambda_{1} \frac{S E}{N}+\lambda_{2} \frac{S I}{N}+\lambda_{3} \frac{S R}{N}-\delta E\right)+\frac{\lambda_{2}+\frac{\varepsilon}{d} \lambda_{3}}{\omega}(\gamma E-\omega I)+\frac{\lambda_{3}}{d}(\varepsilon I-d R) \\
=\left(\lambda_{1} E+\lambda_{2} I+\lambda_{3} R\right)\left(R_{0} \frac{S}{N}-1\right) \leq\left(\lambda_{1} E+\lambda_{2} I+\lambda_{3} R\right)\left(R_{0}-1\right) .
\end{gathered}
$$

Furthermore, $V^{\prime}=0$ if and only if $E=I=R=0$ or $R_{0}=1$. Therefore the maximum compact invariant set in $\left\{(S, E, I, R) \in \mathrm{R}_{+}^{4} \mid V^{\prime}=0\right\}$ is the singleton $E_{0}$ when $R_{0} \leq 1$. LaSalle's invariance principle then implies that $E_{0}$ is globally asymptotically stable in $\Gamma$.

\section{Local Stability of the Endemic Equilibrium}

Theorem 2. The endemic equilibrium $E^{*}$ of (1) is locally asymptotically stable if $R_{0}>1$.

Proof. The Jacobian matrix of (1) at a point $E^{*}$ is 


$$
J\left(E^{*}\right)=\left(\begin{array}{cccc}
a_{1} & a_{2} & a_{3} & a_{4} \\
a_{5} & a_{2} & a_{3} & a_{4} \\
0 & \gamma & -\omega & 0 \\
0 & 0 & \varepsilon & -d
\end{array}\right) .
$$

where $a_{1}=-\frac{N^{*}-S^{*}}{N^{* 2}} m-d, a_{2}=-\frac{S^{*}\left(\lambda_{1} N^{*}-m\right)}{N^{* 2}}, a_{3}=-\frac{S^{*}\left(\lambda_{2} N^{*}-m\right)}{N^{* 2}}, a_{4}=-\frac{S^{*}\left(\lambda_{3} N^{*}-m\right)}{N^{* 2}}$, $a_{5}=\frac{N^{*}-S^{*}}{N^{* 2}} m, \quad m=\lambda_{1} E^{*}+\lambda_{2} I^{*}+\lambda_{3} R^{*}$.

Its characteristic equation is $\operatorname{det}\left(\lambda I-J\left(E^{*}\right)\right)=0$, where $I$ is the unit matrix, $S^{*}=\delta E^{*} N^{*} / m$, $E^{*}=\omega I^{*} / \gamma \quad, \quad R^{*}=\varepsilon I^{*} / d \quad$. So the characteristic equation become to $(\lambda+d)\left(\lambda^{3}+b_{1} \lambda^{2}+b_{2} \lambda+b_{3}-\varepsilon \gamma a_{4}\right)=0$, where

$$
\begin{gathered}
b_{1}=\omega+d+\frac{m}{N^{*}}+\frac{\left(\lambda_{2} d+\lambda_{3} \varepsilon\right) \delta \gamma}{\lambda_{1} d \omega+\left(\lambda_{2} d+\lambda_{3} \varepsilon\right) \gamma}>0, b_{3}=\frac{m S^{*}(\gamma d+\varepsilon \gamma+\omega d)}{N^{* 2}}+\omega \delta m \frac{N^{*}-S^{*}}{N^{* 2}}>0, \\
b_{2}=d \omega+\frac{\left(\lambda_{2} d+\lambda_{3} \varepsilon\right) \delta \gamma d}{\lambda_{1} d \omega+\left(\lambda_{2} d+\lambda_{3} \varepsilon\right) \gamma}+\delta m \frac{N^{*}-S^{*}}{N^{* 2}}+\frac{\omega N^{*}+(d+\gamma) S^{*}}{N^{* 2}} m>0 .
\end{gathered}
$$

It is easy to calculate $b_{1} b_{2}-\left(b_{3}+\varepsilon \gamma a_{4}\right)>0$. According to Hurwitz criterion, the endemic equilibrium $E^{*}$ of (1) is local asymptotical stability.

\section{Global Stability of the Endemic Equilibrium}

Let $\tau=d t$, the system (1) becomes

$$
\left\{\begin{array}{l}
\frac{d S}{d \tau}=\frac{A}{d}-\beta_{1} \frac{S E}{N}-\beta_{2} \frac{S I}{N}-\beta_{3} \frac{S R}{N}-S, \\
\frac{d E}{d \tau}=\beta_{1} \frac{S E}{N}+\beta_{2} \frac{S I}{N}+\beta_{3} \frac{S R}{N}-\delta E, \\
\frac{d I}{d \tau}=\gamma_{0} E-\omega I, \\
\frac{d R}{d \tau}=\varepsilon_{0} I-R .
\end{array}\right.
$$

where $\beta_{1}=\lambda_{1} / d, \quad \beta_{2}=\lambda_{2} / d, \quad \beta_{3}=\lambda_{3} / d, \quad \delta=1+\gamma_{0}+\alpha_{10}+k_{10}, \omega=1+\varepsilon_{0}+\alpha_{20}+k_{20}, \gamma_{0}=\gamma / d$, $\varepsilon_{0}=\varepsilon / d, \alpha_{10}=\alpha_{1} / d, \alpha_{20}=\alpha_{2} / d, k_{10}=k_{1} / d, k_{20}=k_{2} / d$. The equation for the total population is

$$
\frac{d N}{d \tau}=\frac{A}{d}-N-\left(\alpha_{10}+k_{10}\right) E-\left(\alpha_{20}+k_{20}\right) I
$$


We use $N$ as a variable in place of the variable $S$ to give the following system

$$
\left\{\begin{array}{l}
\frac{d E}{d \tau}=\left(\beta_{1} \frac{E}{N}+\beta_{2} \frac{I}{N}+\beta_{3} \frac{R}{N}\right)(N-E-I-R)-\delta E, \\
\frac{d I}{d \tau}=\gamma_{0} E-\omega I, \\
\frac{d R}{d \tau}=\varepsilon_{0} I-R, \\
\frac{d N}{d \tau}=\frac{A}{d}-N-\left(\alpha_{10}+k_{10}\right) E-\left(\alpha_{20}+k_{20}\right) I .
\end{array}\right.
$$

The system (4) is equivalent to (3). From biological considerations, we discuss (4) in the closed set $T=\left\{(E, I, R, N) \in R_{+}^{4} \mid 0 \leq E+I+R \leq N \leq A / d\right\}$. We denote by $\partial T$ and $\dot{T}$ the boundary and the interior of $T$ in $R_{+}^{4}$, respectively.

For (4), the global stability of the endemic equilibrium $E^{*}$ is considered when $\alpha_{10}=\alpha_{20}=k_{10}=k_{20}=0$. Since $N \rightarrow A / d$ as $\tau \rightarrow \infty$, we can obtain the following limit system

$$
\left\{\begin{array}{l}
\frac{d E}{d \tau}=\left(\beta_{1} E+\beta_{2} I+\beta_{3} R\right) \frac{d}{A}\left(\frac{A}{d}-E-I-R\right)-\delta E \\
\frac{d I}{d \tau}=\gamma_{0} E-\omega I \\
\frac{d R}{d \tau}=\varepsilon_{0} I-R
\end{array}\right.
$$

We make the change of variable $x=A / d-E-I-R, y=E, z=I$, then the following (5) is equivalent to the above system

$$
\left\{\begin{array}{l}
\frac{d x}{d \tau}=\frac{A}{d}-x-\left(\beta_{3} \frac{A}{d}+\left(\beta_{1}-\beta_{3}\right) y+\left(\beta_{2}-\beta_{3}\right) z-\beta_{3} x\right) \frac{d}{A} x, \\
\frac{d y}{d \tau}=\left(\beta_{3} \frac{A}{d}+\left(\beta_{1}-\beta_{3}\right) y+\left(\beta_{2}-\beta_{3}\right) z-\beta_{3} x\right) \frac{d}{A} x-\delta y \\
\frac{d z}{d \tau}=\gamma_{0} y-\omega z .
\end{array}\right.
$$

Theorem 3. Consider the following system

$$
\dot{x}=f(x), f \in C^{1}\left(R^{n}\right), \quad x \in T \subset R^{n} .
$$

where $T$ is an open set, if the system (6) satisfies the following conditions: (1) The system (6) exists a compact absorbing set $K \subset T$ and has a unique equilibrium $P$ in $\dot{T}$; (2) $P$ is local asymptotically 
stable; (3) The system (6) satisfies a Poincare-Bendixson criterion; (4) A periodic orbit of (6) is asymptotically orbitally stable. Then the only equilibrium $P$ is the globally asymptotically stable in $T$.

Theorem 4. A sufficient condition for a periodic orbit $P=\{P(t): 0 \leq t \leq \tau\}$ of (6) to be asymptotically orbitally stable with asymptotic phase is that the linear system $z^{\prime}(t)=\frac{\partial f^{[2]}}{\partial t}(P(t)) z(t)$ is asymptotically stable, where $\frac{\partial f^{[2]}}{\partial t}$ is the second additive compound matrix of the Jacobian matrix $\frac{\partial f}{\partial t}$ of $f$. The system (6) called the second compound system of the orbit $P(t)$.

Lemma 2. Any periodic solution to (5), if it exists, is asymptotically orbitally stable.

Proof. Suppose that the solution $(x(t), y(t), z(t))$ is periodic of least period $\tau>0$ such that $(x(0), y(0), z(0)) \in \dot{T}$. The periodic orbit is $P=\{P(t): 0 \leq t \leq \tau\}$. We have the second compound system $y^{\prime}=J(P) y$ of the differential system $x^{\prime}=J^{[2]}(P) x$ in the periodic solution is the following periodic linear system

$$
\left\{\begin{array}{l}
X^{\prime}=a_{11} X+\left(\beta_{2}-\beta_{3}\right) \frac{d}{A} x(Y+Z) \\
Y^{\prime}=\gamma_{0} X+a_{22} Y-\left(\beta_{1}-\beta_{3}\right) \frac{d}{A} x Z \\
Z^{\prime}=a_{32} Y-\left(\delta+\omega-\left(\beta_{1}-\beta_{3}\right) \frac{d}{A} x\right) Z
\end{array}\right.
$$

where

$$
\begin{gathered}
a_{11}=-1+\beta_{3} \frac{d}{A} x-\left(\beta_{3} \frac{d}{A}+\left(\beta_{1}-\beta_{3}\right) y+\left(\beta_{2}-\beta_{3}\right) z-\beta_{3} x\right) \frac{d}{A}-\delta, \\
a_{22}=-1-\omega+\beta_{3} \frac{d}{A} x-\left(\beta_{3} \frac{d}{A}+\left(\beta_{1}-\beta_{3}\right) y+\left(\beta_{2}-\beta_{3}\right) z-\beta_{3} x\right) \frac{d}{A}, \\
a_{32}=-2 \beta_{3} \frac{d}{A} x+\left(\beta_{3} \frac{d}{A}+\left(\beta_{1}-\beta_{3}\right) y+\left(\beta_{2}-\beta_{3}\right) z\right) \frac{d}{A} \\
J(P)=\left(\begin{array}{ccc}
a_{11}+\delta & -\left(\beta_{1}-\beta_{3}\right) \frac{d}{A} x & -\left(\beta_{2}-\beta_{3}\right) \frac{d}{A} x \\
a_{32} & \left(\beta_{1}-\beta_{3}\right) \frac{d}{A} x-\delta & \left(\beta_{2}-\beta_{3}\right) \frac{d}{A} x \\
0 & \delta & -\omega
\end{array}\right),
\end{gathered}
$$




$$
J^{[2]}(P)=\left(\begin{array}{ccc}
a_{11} & \left(\beta_{2}-\beta_{3}\right) \frac{d}{A} x & \left(\beta_{2}-\beta_{3}\right) \frac{d}{A} x \\
\gamma_{0} & a_{22} & -\left(\beta_{1}-\beta_{3}\right) \frac{d}{A} x \\
0 & a_{32} & -\delta-\omega+\left(\beta_{1}-\beta_{3}\right) \frac{d}{A} x
\end{array}\right) .
$$

Suppose that $(X(t), Y(t), Z(t))$ is a solution to (7). Let $V(X, Y, Z, x, y, z)=\sup \{|X|, y(|Y|+|Z|) / z\}$. From the condition (1) of Theorem 3, we can know there exists constant $\eta>0$ such that $V(X, Y, Z, x, y, z) \geq \eta|(X, Y, Z)|, \forall(X, Y, Z) \in R^{3},(x, y, z) \in P$. Direct calculations lead to the following differential inequalities

$$
\begin{gathered}
D_{+}|X(t)| \leq a_{11}|X(t)|+\left(\beta_{2}-\beta_{3}\right) x d / A(|Y(t)|+|Z(t)|), \\
D_{+}|Y(t)| \leq \gamma_{0}|X(t)|+a_{22}|Y(t)|-\left(\beta_{1}-\beta_{3}\right) d x / A|Z(t)|, \\
D_{+}|Z(t)| \leq a_{32}|Y(t)|-\left(\delta+\omega-\left(\left(\beta_{1}-\beta_{3}\right) d x / A\right)\right)|Z(t)| .
\end{gathered}
$$

Using (9) and (10), having

$$
D_{+} y / z(|Y(t)|+|Z(t)|)=|X(t)| y \gamma_{0} / z+\left(y^{\prime} / y-z^{\prime} / z-\omega-1\right)(|Y(t)|+|Z(t)|) y / z
$$

From (6) and (9) leading to

$$
D_{+} V(t) \leq \sup \left\{g_{1}, g_{2}\right\} V(t)
$$

where

$$
\begin{gathered}
g_{1}=-1-\delta-\left(\beta_{3} d / A-\beta_{3} d x / A+\left(\beta_{1}-\beta_{3}\right) y+\left(\beta_{2}-\beta_{3}\right) z-\beta_{3} x\right) d / A+\left(\beta_{2}-\beta_{3}\right) x d z / y A \\
g_{2}=y \gamma_{0} / z+\left(y^{\prime} / y-z^{\prime} / z-\omega-1\right) .
\end{gathered}
$$

Rewriting the last two equations of (5), obtaining

$$
\begin{gathered}
\left(\beta_{2}-\beta_{3}\right) d x z / A y=y^{\prime} / y+\delta-\beta_{3} d x / A y-\left(\beta_{1}-\beta_{3}\right) d x / A+\beta_{3} d x^{2} / A y \\
y \gamma_{0} / z=\omega+z^{\prime} / z
\end{gathered}
$$

Substituting (15) into (13) and (16) into (14), having 


$$
\begin{gathered}
g_{1}=y^{\prime} / y-1-\beta_{3} d / A(d / A-x-y-z)-\beta_{3} d x / A(1-x-y)-\beta_{1} d y / A-\beta_{2} d z / A, \\
g_{2}=y^{\prime} / y-1 .
\end{gathered}
$$

Thus $\sup \left\{g_{1}(t), g_{2}(t)\right\} \leq y^{\prime} / y-1, \int_{0}^{\tau} \sup \left\{g_{1}(t), g_{2}(t)\right\} d t \leq\left.\ln y(t)\right|_{0} ^{\tau}-\tau=-\tau$. From (10), we obtain $\lim _{t \rightarrow \infty} V(t)=0$ and in turn that $V(X, Y, Z, x, y, z) \geq \eta|(X, Y, Z)|$ as $t \rightarrow \infty$. As a result, the second compound system (5) is asymptotically stable and the periodic solution $(x(t), y(t), z(t))$ is asymptotical orbital stability by Theorem 3 .

Lemma 3. The system (5) is uniformly persistent when $R_{0}>1$.

Proof. Set $G=\left\{P_{0}\right\}$, when $R_{0}>1$, the stable set $G^{s}$ is just contained in the S-axis and thus in the boundary of $\Gamma$. It also implies that the stable set $G^{s}$ is isolated in $\Gamma$. Then, when $R_{0}>1$, the system (5) satisfies the conditions of Theorem 2 of [18], namely, (a) the maximal compact invariant set $G$ in the boundary of $\Gamma$ is isolated and (b)the stable set $G^{s}$ of $G$ is contained in the boundary of $\Gamma$. Therefore, the system (5) is uniformly persistent in $\Gamma$ when $R_{0}>1$.

Lemma 4. The system (3) is competitive when $R_{0}>1$ and $\alpha_{10}=\alpha_{20}=k_{10}=k_{20}=0$.

Proof. Set $x_{1}=S, x_{2}=E, x_{3}=I, x_{4}=R$, the system (3) is replaced by

$$
\left\{\begin{array}{l}
x_{1}^{\prime}=\frac{A}{d}-\beta_{1} S \frac{d}{A}\left(\frac{A}{d}-x_{1}-x_{3}-x_{4}\right)-\beta_{2} S \frac{d}{A}\left(\frac{A}{d}-x_{1}-x_{2}-x_{4}\right)-\beta_{3} S \frac{d}{A}\left(\frac{A}{d}-x_{1}-x_{2}-x_{3}\right)-x_{1}, \\
x_{2}{ }^{\prime}=\beta_{1} S \frac{d}{A} x_{2}+\beta_{2} S \frac{d}{A} x_{3}+\beta_{3} S \frac{d}{A} x_{4}-\delta x_{2}, \\
x_{3}{ }^{\prime}=\gamma_{0} x_{2}-\omega x_{3}, \\
x_{4}{ }^{\prime}=\varepsilon_{0} x_{3}-x_{4} .
\end{array}\right.
$$

Furthermore, the system has the following form

$$
x^{\prime}=(A(t)-I) \mathrm{x}+C(t)
$$

where $x=\left(x_{1}, x_{2}, x_{3}, x_{4}\right) \in R^{4}, I$ denotes the $4 \times 4$ unit matrix, $C(t)$ is a vector function, and

$$
A(t)=\left(\begin{array}{cccc}
\left(\beta_{1}+\beta_{2}+\beta_{3}\right) S \frac{d}{A} & \left(\beta_{2}+\beta_{3}\right) S \frac{d}{A} & \left(\beta_{1}+\beta_{3}\right) S \frac{d}{A} & \left(\beta_{1}+\beta_{2}\right) S \frac{d}{A} \\
0 & \beta_{1} S \frac{d}{A}-\gamma_{0} & \beta_{2} S \frac{d}{A} & \beta_{3} S \frac{d}{A} \\
0 & \gamma_{0} & -\varepsilon_{0} & 0 \\
0 & 0 & \varepsilon_{0} & 0
\end{array}\right) .
$$

The off-diagonal entries in this matrix are non-negative, the system as a whole is quasimonotone. Thus it 
can be verified that the system (3) is competitive with respect to the partial ordering defined by the orthant $K=\left\{(S, E, I, R) \in R^{4} \mid S \geq 0, E \geq 0, I \geq 0, R \geq 0\right\}$.

Theorem 5. If $R_{0}>1$, the endemic equilibrium $E^{*}$ of (1) is globally asymptotically stable when $\alpha_{1}=\alpha_{2}=k_{1}=k_{2}=0$.

\section{The Numerical Simulation}
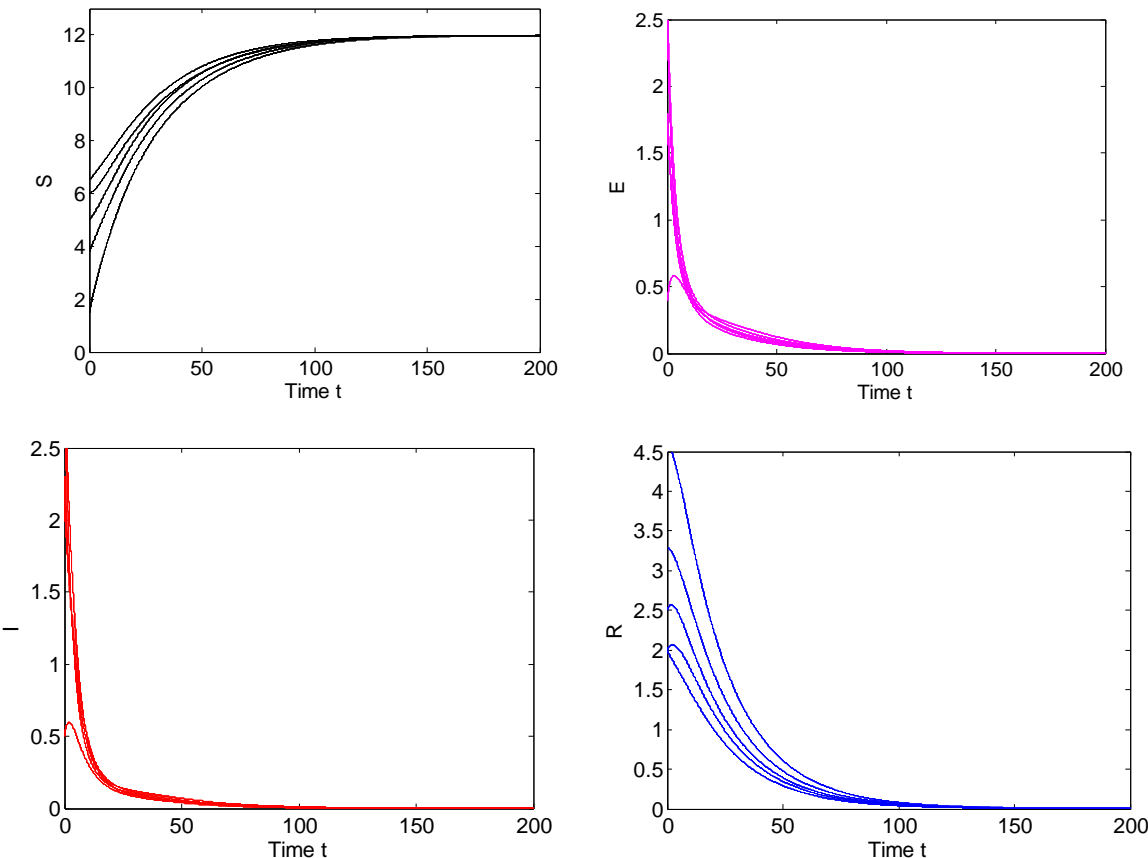

Fig. 1. Variational curves of $S 、 E 、 I$ and $R$ with $t$ when $R_{0}=0.55$
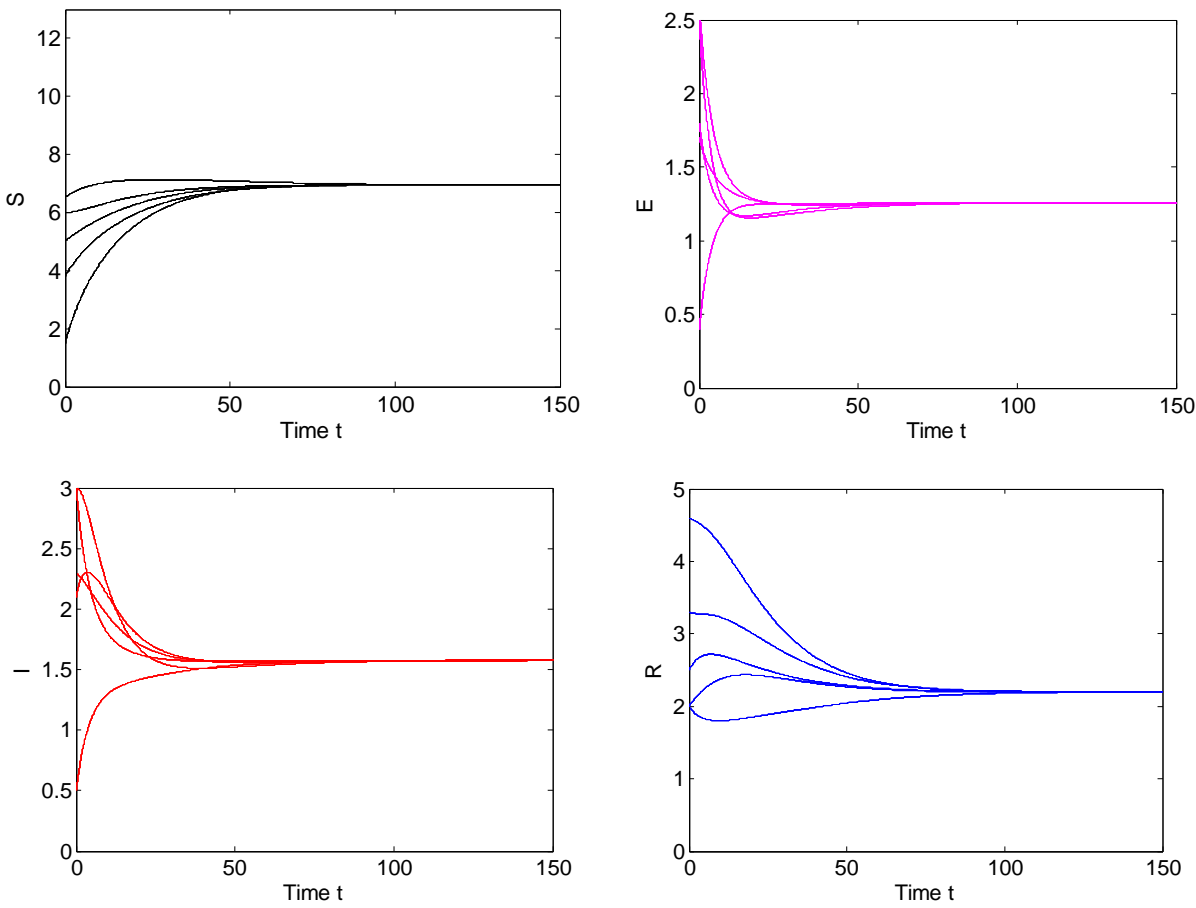

Fig. 2. Variational curves of $S 、 E 、 I$ and $R$ with $t$ when $R_{0}=1.73$. 
Set the parameters $A=0.6, d=0.05, \varepsilon=0.07, \gamma=0.15, \alpha_{1}=0.08, \alpha_{2}=0.1, k_{1}=0.04$, $k_{2}=0.08$, And set five initial values $(1.5,2.5,3,4.6),(6,0.4,3,2.5),(6.5,1.8,0.5,2)$, $(3.8,2.6,2.1,3.3),(5,1.7,2.3,2)$.

When set $\lambda_{1}=0.08, \lambda_{2}=0.15$ and $\lambda_{3}=0.03$, the basic reproduction number $R_{0}=0.55<1$. The disease-free equilibrium $E_{0}=(12,0,0,0)$ is global asymptotical stability in $\Gamma$, as shown in the follow Fig. 1.

When set $\lambda_{1}=0.07, \lambda_{2}=0.15$ and $\lambda_{3}=0.05$, the basic reproduction number $R_{0}=1.73>1$. The endemic equilibrium $E^{*}=(6.958,1.261,1.576,2.203)$ is global asymptotical stability in $\Gamma$, as shown in the Fig. 2.

\section{Conclusions}

This paper has discussed an SEIR epidemic model with infectious force in latent and recovered period. The basic reproductive number $R_{0}$ is identified. If $R_{0}<1$, the disease-free equilibrium $E_{0}$ is globally asymptotically stable in the feasible region so that the disease dies out. If $R_{0}>1$, the endemic equilibrium $E^{*}$ is globally asymptotically stable in the interior of the feasible region, and once the disease appears, it eventually persists at the unique endemic equilibrium level. It is obtained that $R_{0}$ is a monotony increase function of $\lambda_{1}$ and $\lambda_{3}$ by the formula of $R_{0}$. The smaller the infectious force in latent and recovered period, the more detrimental to the control and elimination of the infectious disease. Therefore, the infected patients, the latent patients and recovered patients are all controlled for the disease with the infectious force in latent and recovered period.

In this paper, we study the asymptotic stability of the SEIR model with infectious force in latent and recovered period, and enriches the research work of the dynamics of infectious diseases. In addition, there is a lot of work waiting for us to study in this field. It is difficult for the pulse mode of the epidemic model to be thought of. We will study these problems in the future.

\section{Acknowledgment}

This work is supported by the Natural Science Foundation of Anhui (KJ2015A331) and the Natural Science Foundation of Anhui (KJ2015A308).

\section{References}

[1] Wang, J., Zhang, Z., \& Jin, Z. (2010). Analysis of an sir model with bilinear incidence rate. Nonlinear Analysis: Real World Applications, 11(4), 2390-2402.

[2] Kandhway, K., \& Kuri, J. (2014). How to run a campaign: Optimal control of SIS and SIR information epidemics. Applied Mathematics and Computation, 231(13), 79-92.

[3] Muroya, Y., Huaixing, L., \& Kuniya, T. (2014). Complete global analysis of an SIRS epidemic model with graded cure and incomplete recovery rates. Journal of Mathematical Analysis and Applications, 410(2), 719-732.

[4] Yanan, Z., \& Daqing, J. (2014). The threshold of a stochastic SIRS epidemic model with saturated incidence. Applied Mathematics Letters, 34(1), 90-93.

[5] Qun, L., Qingmei, C., \& Daqing, J. (2015). The threshold of a stochastic delayed SIR epidemic model with 
temporary immunity. Physica A: Statistical Mechanics and its Applications, 450(15), 115-125.

[6] Zengyun, H., Zhidong, T., \& Long, Z. (2014). Stability and bifurcation analysis in a discrete SIR epidemic model. Mathematics and Computers in Simulation, 97(2), 80-93.

[7] Rui, X., \& Yanke, D. (2011). A delayed SIR epidemic model with saturation incidence and a constant infectious period. Journal of Applied Mathematics and Computing, 35(1-2), 229-250.

[8] Zhixing, H., Wanbiao, M., \& Shigui, R. (2012). Analysis of SIR epidemic models with nonlinear incidence rate and treatment. Mathematical Biosciences, 238(1), 12-20.

[9] Qianqian, C., \& Qiang, Z. (2015). Global stability of a discrete SIR epidemic model with vaccination and treatment. Journal of Difference Equations and Applications, 21(2), 111-117.

[10] Zhichao, J., \& Wanbiao, M. (2015). Permanence of a delayed SIR epidemic model with general nonlinear incidence rate. Mathematical Methods in the Applied Sciences, 38(3), 505-516.

[11] Zhichao, J., Wanbiao, M., et al. (1999). Global dynamics of a SEIR model with varying total population size. Mathematical Biosciences, 160(2), 191-213.

[12] Meng, F., Michae, 1 Y., \& Wang, K. (2001). Global stability of an SEIS epidemic model with recruitment and a varying total population size. Mathematical Biosciences, 170(2), 199-208.

[13] Chengjun, S., \& Ying-Hen, H. (2010). Global analysis of an SEIR model with varying population size and vaccination. Applied Mathematical Modelling, 34(10), 2685-2697.

[14] Na, Y., Qingling, Z., Kun, M., Dongmei, Y., \& Qin, L. (2009). Analysis and control of an SEIR epidemic system with nonlinear transmission rate. Mathematical and Computer Modelling, 50(9), 1498-1513.

[15] Tailei, Z., \& Zhidong, T. (2008). Global asymptotic stability of a delayed SEIRS epidemic model with saturation incidence. Chaos, Solitons \& Fractals, 37(5), 1456-1468.

[16] Qingling, Z., Chao, L., \& Xue, Z. (2012). Analysis and control of an SEIR epidemic system with nonlinear transmission rate. Complexity, Analysis and Control of Singular Biological Systems, 421(1), 203-225.

[17] Lili, L., Jinliang, W., \& Xianning, L. (2015). Global stability of an SEIR epidemic model with age-dependent latency and relapse. Nonlinear Analysis: Real World Applications, 24(1), 18-35.

[18] Michael, Y., Graef, R., Liancheng, W., et al. (1999). Global dynamics of a SEIR model with varying total population size. Mathematical Biosciences, 160(2), 191-213.

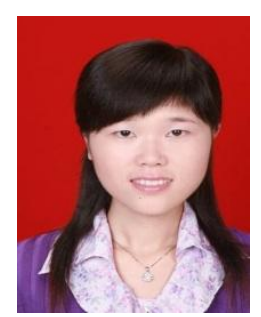

Yanli Ma was born in Anhui of China on October 25, 1983. She finished her bachelor's degree in mathematics and computing science from Huaibei Normal University, the Department of Mathematics, Huaibei, Anhui, China in 2006. She finished her master's degree in applied mathematics from Xi'an Jiao Tong University, College of Science, Xian, Shanxi, China in 2009. Her research interest lies in applied mathematics, biology mathematics, dynamics of infectious diseases and computer simulation. So far in 2009, she is working as a teacher at department of common course, Anhui Xinhua University, Hefei Anhui, China. She has published several papers in national Journals. Her current research interest lies in the study of differential dynamic system. 